\title{
ENRAIZAMENTO DE ESTACAS DE CANA-DO-BREJO $\left({ }^{1}\right)$
}

\author{
CLÁUDIA PFALTZGRAFF MARIOTTO FERREIRA DE AZEVEDO $\left({ }^{2}\right)$; PAULO CESAR FERREIRA $\left({ }^{2}\right)$; \\ JOAQUIM SIQUEIRA DOS SANTOS $\left({ }^{3}\right)$; LILIANA AUXILIADORA AVELAR PEREIRA PASIN $\left({ }^{*}\right)$
}

\begin{abstract}
RESUMO
A propagação vegetativa de espécies medicinais vem despertando o interesse de pesquisas agronômicas já que se constitui em ferramenta básica para qualquer cultivo em escala comercial. Neste contexto, este trabalho objetivou avaliar a propagação por estaquia da cana-do-brejo, considerando o tipo de estaca, a presença de folhas e o tipo de substrato no enraizamento. Foram utilizados dois tipos de substrato: areia e um composto 3:2:1 (terra, areia e húmus). Foram utilizadas estacas da porção apical e mediana, com folhas inteiras, meia folha e sem folha. O delineamento experimental foi inteiramente casualizado, com 10 repetições e esquema fatorial $3 \times 2 \times 2$. Aos 60 dias foram avaliados a porcentagem de sobrevivência das estacas, o número de raízes, número de brotos, comprimento de maior raiz e a biomassa seca das raízes. Foram verificados o maior enraizamento e maior número de brotos nas estacas apicais com folha e meia-folha. Nas estacas medianas e apicais sem folha não se observou enraizamento efetivo nos substratos utilizados.
\end{abstract}

Palavras-chave: estaquia, Costus spicatus, substrato, tipo de estaca.

\section{ABSTRACT \\ ROOTING OF CUTTINGS OF SPIRAL GINGERS (Costus spicatus Jacq.)}

The vegetative propagation of medicinal species is becoming a basic tool for any cultivation in commercial scale. In this context, this work aimed to evaluate the propagation of spiral gingers cuttings, considering the type of cutting, presence of leaves and type of substrate for rooting. Two types of substrate were used: sand and a composition 3:2:1 (land, sand and humus). Cuttings of the apical and medium portion were tested, with either intact leaves, half leaf or without leaf, using a completely randomized design with 10 replications. The percentage of cutting survival, number of roots, number of buds, length of larger root as well as root dry biomass were evaluated within 60 days. The best rooting and number of buds were observed using the apical cutting with intact and half leaf. The medium and apical cuttings without leaf did not exhibited effective rooting in the tested substrates.

Key words: Costus spicatus, substrate, rooting, cutting.

${ }^{1}$ ) Recebido para publicação em 16 de outubro de 2007 e aceito em 10 de junho de 2009.

$\left(^{2}\right)$ Graduandos do Curso de Ciências Biológicas. UNIVAP - Universidade do Vale do Paraíba - Av. Shishima Hifumi 2911, 12244000 São José dos Campos (SP). E-mail: claumariotto@hotmail.com; pecefe.cesar@yahoo.com.br

$\left(^{3}\right)$ Setor de Botânica. UNIVAP - Universidade do Vale do Paraíba.

$\left({ }^{4}\right)$ Faculdade de Educação. UNIVAP - Universidade do Vale do Paraíba. Doutora em Agronomia. E-mail. lpasin@univap.br (*) Autora correspondente. 


\section{INTRODUÇÃO}

A cana-do-brejo (Costus spicatus Jacq.), espécie pertencente à família Zingiberaceae, também denominada cana-branca ou cana-do-mato, é uma planta fitoterápica, nativa em quase todo o Brasil, principalmente na Mata Atlântica e região Amazônica (Silva Junior, 1998). Caracteriza-se por ser uma planta perene, rizomatosa, não ramificada, ereta, que pode atingir $2 \mathrm{~m}$ de altura. A planta é utilizada na medicina popular, principalmente na região Amazônica; sua ação é depurativa e diurética, aliviando infecções urinárias e auxiliando na eliminação de pedras renais (LORENZI e MATOS, 2002).

Trabalhos sobre a forma mais efetiva de propagação e cultivo de plantas medicinais são incipientes ou inexistentes para a maioria das espécies, sendo, portanto, necessário o desenvolvimento de estudos relacionados à sua propagação, principalmente em função do aumento da demanda por parte das indústrias farmacêuticas e cosmética.

Dentre os métodos de propagação vegetativa, a estaquia é ainda a técnica de maior viabilidade econômica para o estabelecimento de plantios clonais, pois permite, a um menor custo, a multiplicação de genótipos selecionados em um curto período de tempo (Momenté et al., 2002).

O interesse da pesquisa na propagação vegetativa de plantas medicinais é bastante recente e dentre as espécies já estudadas tem-se: Lippia alba (BIAsI e COSTA, 2003), Ocimum gratissimum (EHLert et al., 2004), Baccharis articulata, Baccharis trimera e Baccharis stenocephala (BonA et al., 2005a, b).

O sucesso da propagação vegetativa sofre a influência de vários fatores, entre eles a posição da estaca no ramo, o grau de lignificação, a quantidade de reservas e diferenciação dos tecidos, presença ou ausência de folhas nas estacas, espécie, cultivar, época de coleta e tipo de substrato.

Diversos estudos demonstram que a utilização de estacas herbáceas, semilenhosas e lenhosas, com presença ou ausência de folhas, assim como a época da coleta das estacas, têm grande influência no seu enraizamento (МотA et al., 2002).

A presença de folhas garante a sobrevivência das estacas, tanto pela síntese de carboidratos através da fotossíntese, como pelo fornecimento de auxinas e outras substâncias importantes no processo de formação de raízes, estimulando a atividade cambial e a diferenciação celular (MOMENTÉ et al., 2002).

O substrato é fundamental para o desenvolvimento das raízes nas estacas, devendo possuir baixa densidade, boa aeração e drenagem para evitar o acúmulo de umidade, devendo estar isento de pragas e substâncias tóxicas (KAMPF, 2000; WeNDLING et al., 2002).

A condição fisiológica das estacas também tem papel relevante no enraizamento, visto que ao longo do ramo, há variação no conteúdo de carboidratos e de substâncias promotoras e inibidoras do crescimento nos tecidos, e as estacas obtidas de diferentes porções do ramo tendem a diferir quanto ao potencial de enraizamento (EHLERT et al., 2004).

Considerando que estudos com a propagação de cana-do-brejo são inexistentes, este trabalho objetiva avaliar a influência da posição da estaca no ramo, tipo de substrato e presença de folhas na propagação por estaquia.

\section{MATERIAL E MÉTODOS}

O trabalho foi desenvolvido em telado, localizado em São José dos Campos (SP). Os ramos da cana-do-brejo utilizados foram coletados de plantas adultas, cultivadas nos canteiros de plantas medicinais da UNIVAP.

Foram utilizadas estacas da porção apical e mediana, com aproximadamente $15 \mathrm{~cm}$. O meristema apical foi mantido nas estacas apicais. Para as duas posições das estacas, foram utilizadas estacas com duas folhas inteiras, duas folhas cortadas ao meio e sem folhas. Após preparadas, as estacas foram acondicionadas em sacos plásticos individuais, contendo areia ou um composto 3:2:1 (terra, areia e húmus) e transferidas para um telado com nebulização intermitente.

O delineamento experimental utilizado foi o inteiramente casualizado, com 10 repetições. Os tratamentos avaliados foram dispostos no esquema fatorial $3 \times 2 \times 2$, constituído dos seguintes fatores: presença de folhas (folha inteira, meia-folha e sem folha), posição das estacas (apical e mediana) e substratos (areia e composto).

A avaliação foi realizada 60 dias após a instalação do experimento, avaliando-se as variáveis: porcentagem de enraizamento, número de estacas com brotação, número de raízes, comprimento da maior raiz e biomassa seca das raízes por estaca.

As raízes provenientes de cada estaca foram acondicionadas separadamente em sacos de papel, os quais foram dispostos em estufa C.B. retilínea, à temperatura média de $50^{\circ} \mathrm{C}$, até a obtenção de massa constante, atingido em aproximadamente 48 horas, para determinação da matéria seca do sistema radicular. 
Os resultados foram submetidos à análise de variância, e em caso de teste F significativo, as médias foram comparadas pelo teste Tukey a 5\% de probabilidade.

\section{RESULTADOS E DISCUSSÃO}

A análise de variância revelou efeito significativo da posição da estaca e da presença de folhas sobre as variáveis porcentagem de enraizamento, número de estacas com brotação, número de raízes, comprimento da maior raiz e biomassa seca das raízes por estaca. A interação posição $\mathrm{x}$ presença de folhas também foi significativa; entretanto, o mesmo não ocorreu com o fator substrato e suas interações.
Observou-se que as estacas apicais com folha e meia-folha, tinham maior número, maior comprimento de raiz, maior biomassa seca e maior número de brotos (Tabela 1). A presença da folha em estacas semilenhosas, é essencial para a formação de novas raízes, já que nas folhas ocorre a produção de carboidratos pela fotossíntese, além de auxinas e outras substâncias necessárias para o enraizamento (HARTMANN et al., 1997). Esse efeito benéfico pode ser observado no enraizamento de cipó-mil-homens, que enraizou $69 \%$ em estacas com folhas inteiras e $46 \%$ em estacas com meia folha (CORRÊA e BIASI, 2003). Estacas de guaco com folha tiveram um enraizamento de $83 \%$, contra $59 \%$ nas estacas sem folha (МотA et al., 2002), evidenciando que a presença de folhas é um fator relevante na obtenção efetiva da propagação por estaca para algumas espécies.

Tabela 1. Média do número de raízes, comprimento da maior raiz, biomassa seca das raízes e número de brotos em estacas de Costus spicatus, nos substratos composto e areia em função da posição e presença de folhas

\begin{tabular}{|c|c|c|c|c|c|c|c|c|}
\hline \multirow{2}{*}{ Folhas } & \multicolumn{2}{|c|}{ Raízes } & \multicolumn{2}{|c|}{ Maior raiz } & \multicolumn{2}{|c|}{ Biomassa seca das raízes } & \multicolumn{2}{|c|}{ Brotos } \\
\hline & Apical & Mediana & Apical & Mediana & Apical & Mediana & Apical & Mediana \\
\hline & \multicolumn{2}{|c|}{$-\mathrm{n}^{\mathrm{o}-}$} & $\longrightarrow$ & $\bar{\square}$ & 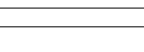 & & $\overline{-1}$ & \\
\hline & \multicolumn{8}{|c|}{ Areia } \\
\hline Com folha & $10,60 \mathrm{~A} \mathrm{a}$ & $4,38 \mathrm{~B} \mathrm{a}$ & $18,90 \mathrm{~A} \mathrm{a}$ & $10,63 \mathrm{~B} \mathrm{a}$ & $70 \mathrm{~A} \mathrm{a}$ & $4 \mathrm{~B} \mathrm{a}$ & $2,50 \mathrm{~A} \mathrm{a}$ & $1,00 \mathrm{~B}$ a \\
\hline Meia folha & $7,71 \mathrm{~A} \mathrm{ab}$ & $3,40 \mathrm{~A} \mathrm{a}$ & $12,14 \mathrm{~A} \mathrm{ab}$ & $9,00 \mathrm{~A} \mathrm{a}$ & $29 \mathrm{~A} \mathrm{ab}$ & $4 \mathrm{~A} \mathrm{a}$ & $2,00 \mathrm{~A} \mathrm{ab}$ & $1,00 \mathrm{~A} \mathrm{a}$ \\
\hline \multirow[t]{2}{*}{ Sem folha } & $2,75 \mathrm{~A} \mathrm{~b}$ & $4,75 \mathrm{~A} \mathrm{a}$ & $6,50 \mathrm{~A} \quad \mathrm{~b}$ & $14,25 \mathrm{~A} \mathrm{a}$ & $4 \mathrm{~A} \mathrm{~b}$ & $4 \mathrm{~A} \mathrm{a}$ & $1,00 \mathrm{~A} \mathrm{~b}$ & $1,14 \mathrm{~A} \mathrm{a}$ \\
\hline & \multicolumn{8}{|c|}{ Composto } \\
\hline Com folha & $9,75 \mathrm{~A} \mathrm{a}$ & $4,29 \mathrm{~B} \mathrm{a}$ & $17,25 \mathrm{~A} \mathrm{a}$ & $12,14 \mathrm{~A} \mathrm{a}$ & $75 \mathrm{~A} \mathrm{a}$ & $4 \mathrm{~B} \mathrm{a}$ & $1,75 \mathrm{~A} \mathrm{ab}$ & $1,56 \mathrm{~A} \mathrm{a}$ \\
\hline Meia folha & $6,75 \mathrm{~A} \mathrm{a}$ & $6,29 \mathrm{~A} \mathrm{a}$ & $12,88 \mathrm{~A} \mathrm{ab}$ & $16,71 \mathrm{~A} \mathrm{a}$ & $13 \mathrm{~A} \mathrm{~b}$ & $4 \mathrm{~A}$ a & $1,63 \mathrm{~A} a b$ & $1,43 \mathrm{~A} \mathrm{a}$ \\
\hline Sem folha & $2,40 \mathrm{~A} \mathrm{~b}$ & $5,00 \mathrm{~A} \mathrm{a}$ & $5,40 \mathrm{~A} \quad \mathrm{~b}$ & $16,11 \mathrm{~B} \mathrm{a}$ & $4 \mathrm{~A} \mathrm{~b}$ & $4 \mathrm{~A}$ a & $1,43 \mathrm{~A} \mathrm{ab}$ & $1,25 \mathrm{~A} \mathrm{a}$ \\
\hline $\mathrm{CV}(\%)$ & 26,4 & 20,8 & 24,8 & 14,10 & 36,4 & 34,3 & 23,3 & 25,6 \\
\hline
\end{tabular}

Médias seguidas pela mesma letra, maiúsculas nas linhas e minúsculas nas colunas, não diferem entre si, a 5\% pelo teste de Tukey.

Com relação à posição das estacas nos ramos, observa-se que a maior taxa de enraizamento pode variar em função da espécie estudada. Em carqueja, o enraizamento diferiu em função da espécie: Baccharis articulata e B. stenocephala tiveram maior taxa de enraizamento das estacas apicais e $B$. trimera nas porções basais e medianas (BONA, 2005a). Do mesmo modo, FACHINELLO et al. (1994) verificaram que, a porção apical em estacas semilenhosas enraíza melhor, pela proximidade dos locais de síntese de auxinas e menor diferenciação dos tecidos. Entretanto, neste estudo a biomassa seca das raízes não diferiu significativamente para as estacas sem folhas, independentemente do tipo de estaca utilizada, evidenciando que a posição da estaca não foi fator determinante para enraizamento efetivo da espécie estudada (Tabela 1).
Todos os exemplares enraizados tiveram brotações aéreas e, em alguns casos, não se verificou formação de raízes na estaca, apenas nos brotos.

Quanto ao número de brotos por estaca, as estacas apicais com folha e meia-folha obtiveram maior número de brotamentos no substrato areia. Entretanto, no composto não houve diferença significativa de brotações entre as porções de estaca. A presença ou ausência de folhas também não diferiu significativamente com relação ao número de brotos quando se utilizou o substrato composto (Tabela 1). Vale ressaltar que, independentemente do substrato utilizado, a presença da folha ou meia-folha foi fator determinante para o enraizamento e a formação de brotos. 
Os tipos de substratos testados não proporcionaram efeito sobre o desenvolvimento das estacas de cana-do-brejo. Não se verificou efeito significativo para número de raízes, tamanho de maior raiz, biomassa seca de raízes, o que evidencia maior capacidade de adaptação às diferentes condições físicas e químicas dos substratos (Costa et al., 2007) e permite a escolha do substrato mais acessível para propagação vegetativa desta espécie.

Com relação à porcentagem de sobrevivência das estacas, apenas detectou-se efeito significativo para as estacas apicais sem folhas, que diferiram nos substratos testados, sendo verificada maior sobrevivência no substrato areia (Tabela 2). Entretanto, este fator não é relevante para a propagação por estaquia da espécie, já que esta diferença não foi detectada nas outras variáveis analisadas.

Tabela 2. Média da porcentagem de sobrevivência das estacas apicais em função dos substratos utilizados em estacas de Costus spicatus

\begin{tabular}{lcc}
\hline \multirow{2}{*}{ Tipo de estaca } & \multicolumn{2}{c}{ Sobrevivência } \\
\cline { 2 - 3 } & Areia & Composto \\
\cline { 2 - 3 } Apical com folha & $100 \mathrm{~A} \mathrm{a}$ & $80 \mathrm{~A} \mathrm{a}$ \\
Apical meia folha & $70 \mathrm{~A} \mathrm{~b}$ & $80 \mathrm{~A} \mathrm{a}$ \\
Apical sem folha & $80 \mathrm{~A} \mathrm{a}$ & $50 \mathrm{~B} \mathrm{~b}$ \\
\hline $\mathrm{CV}(\%)$ & & 11,9 \\
\hline
\end{tabular}

Médias seguidas pela mesma letra, maiúsculas nas linhas e minúsculas nas colunas, não diferem entre si, a 5\% pelo teste de Tukey.

\section{CONCLUSÃO}

Verificou-se desenvolvimento mais efetivo nas estacas apicais com folha e meia-folha, tanto na areia como no composto. As estacas medianas têm menor desenvolvimento do que as apicais, independentemente do substrato utilizado. A porcentagem de sobrevivência é maior nas estacas apicais.

\section{REFERÊNCIAS}

BIASI, L.A.; COSTA, G. Propagação vegetativa de Lippia Alba. Ciência Rural, v.33, p.455-459, 2003.

BONA,C.M.;BIASI, L.A.; ZANETTE,F.;NAKASHIMA,T. Estaquia de três espécies de Baccharis. Ciência Rural, v.35, p.223-226, 2005a.

BONA, C.M.; BIASI, L.A.; ZANETTE, F.; NAKASHIMA, T. Propagação por estaquia de Bacharis articulata (Lam) Pres., Baccharis stenocephala Baker com uso de auxinas. Revista Brasileira de Plantas Medicinais, v.7, p.26-31, 2005b.
CORRÊA, C.F.; BIASI, L.A. Área foliar e tipo de substrato na propagação por estaquia de Cipó-mil-homens. Revista Brasileira de Agrociência, v.9, p.233-236, jul-set, 2003.

COSTA, L.C.B; PINTO, J.E.B.P; BERTOLUCCI, S.K.V. Comprimento da estaca e tipo de substrato na propagação vegetativa de atroveram. Ciência Rural, v.37, p.1157-1160, jul-ago, 2007.

EHLERT, P.A.D.; LUZ, J.M.Q.; INNECCO, R. Propagação vegetativa de alfavaca-cravo utilizando diferentes tipos de estacas e substratos. Horticultura Brasileira, Brasília, v.22, p.1013, 2004.

FACHINELLO, J.C.; HOFFMANN, A.; COSTA NACHTIGAL, J.; KERSTEN, E.; LUCES FORTES, G.R. Propagação de plantas frutíferas de clima temperado. Pelotas: UFPel., 1994, 1179p.

HARTMANN, H.T.; KESTER, D.E.; DAVIES JUNIOR, F.T. Plant propagation: principles and practices. 6.ed. New Jersey: Prentice Hall, 1997. 770p.

KAMPF, A.N. Produção comercial de plantas ornamentais. Guaíba: Agropecuária, 2000. 254p.

LORENZI, H.; MATOS, F.J.A. Plantas medicinais no Brasil. Nova Odessa, SP: Instituto Plantarum, 2002. 544p.

MOMENTÉ, V.G.; BEZERR, A.M.E.; INNECCO, R.; MEDEIROS FILHO, S. Propagação vegetativa por estaquia de mentrasto em diferentes substratos. Revista Ciência Agronômica, v.33, p.5-9, 2002.

MOTA, J.S.; LAZZARINI, G.; LUZ, J.M.Q. Estudo agronômico de plantas medicinais na região de Uberlândia. Revista Eletrônica da Universidade Federal de Uberlândia, Uberlândia 2002.

SILVA JUNIOR, A.A. Plantas medicinais. Florianópolis: EPAGRI: 1998. (CD-ROM)

WENDLING, I.; GATTO, A.; PAIVA, H.N. et al. Substratos, adubação e irrigação na produção de mudas. Viçosa: Aprenda Fácil Editora, 2002. 116p. 